\title{
Electrical characteristics measuring device for security systems
}

\author{
V. $\mathrm{ION}^{1}$, A. GHEORGHE ${ }^{2}$, E. DIACONU ${ }^{2}$ \\ ${ }^{1}$ Valahia University of Targoviste, Doctoral School of Engineering \\ ${ }^{2}$ Valahia University of Targoviste, Faculty of Electrical Engineering, Electronics and Information Technology \\ E-mail: valy_ion@yahoo.com
}

\begin{abstract}
The study aims for the development of a device that is capable to measure and store the electrical characteristics of security systems. The development of the device is done by using the Arduino Mega 2650 board, Arduino Ethernet shield 2, SD card module, 1.8” TFT ISP LCD, keyboard module, ASC712 current sensors, $A . C$ voltage sensors and D.C voltage sensors. The measured data from the current and voltage sensors is stored on the SD card module and sent to the user via the ethernet connection. The data obtained from the device is useful to keep track of the power consumption of any security systems. The device is capable to measure the current and voltage from 10 separate security devices (e.g. cameras, motion sensors, DVRs).
\end{abstract}

Keywords: Arduino, Ethernet, Security Sistems, Sensors.

\section{INTRODUCTION}

Security in the general sense is a primary need of the individual that is extended to different organizational levels. One of the ways to ensure the functions contained in the generic security concept is offered by electronic security systems. As primary functionality, a security system can be defined as a set of devices that detects and signals an intrusion or a state of danger associated with unauthorized entry into the protected space [1]. The development capacity of information processing as well as communication technologies have extended the primary functions of the security system so that, at present, several types of events can be monitored that describe a potentially dangerous situation. The field of application is extremely wide, from residential applications to high security professional systems. Depending on the particularities of the protected objective (with special reference to the values to be protected) the degree of complexity of a system can vary greatly, but the principles underlying an electronic security system are almost always the same. The detection of an event is based on the identification and measurement of some environmental parameters associated with that event. In general, the measurement operation involves an interaction between two systems, one containing the parameter to be measured and the other being the one that performs the measurement operation[4]. The interaction is performed either by direct contact or through a field. Security systems applications currently use electromagnetic waves and elastic waves, but it is possible that technologies based on other principles will be developed in the future. Every device used for the security system consumes more or less power in different scenarios, e.g. the surveillance cameras power consumption is grater in the night time than in the day time because of the built-in infrared system that is used in low light situations [2]. For the measurement of the power consumption of any security system a device that measures and stores the current and voltage consumption is developed. The Arduino Mega 2560 is used for the management and control of the measuring device, the current values are obtained from 10 ASC712 current sensors and the voltage values are obtained from 2 A.C voltage sensor and 8 D.C voltage sensors. The values obtained from the sensors are stored on a SD card using an external SD card module and then sent via the ethernet connection for viewing the data in real time. The device also contains a 1.8" TFT ISP LCD display and a keyboard module that is used for the initial setup and configuration [3].

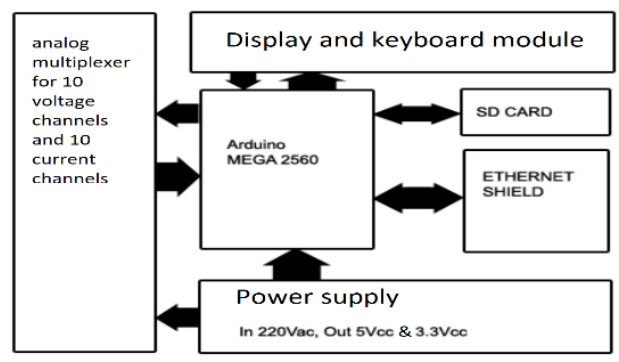

Figure 1 System block diagram.

In Fig.1 we have the block diagram of the measurement device. The values for the current and voltage sensors are received by the Arduino MEGA 2560 developing board then the Arduino MEGA stores the information on a SD card using the external SD card module and sends it via the Ethernet shield to the user for viewing the data in real time. The device is powered by a A.C to D.C power supply that delivers an output of $5 \mathrm{~V}$ and $3 \mathrm{~V}$. The display and the keyboard module is used to configure the device.

\subsection{Arduino Mega 2650}

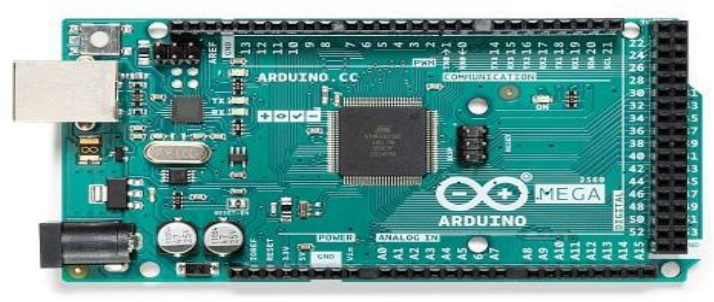

Figure 2 Arduino Mega 2650 developing board. 
The Arduino Mega 2650 development board (e.g. Fig 2) is used for more complex projects. The ATmega2560 microchip is used for the developing board, the microchip is a 8-bit low-power AVR CPU [5]. The development board has 70 pins that are used for input and output, 54 of the pins (14 pins are PWM output pins) are digital pins and 16 pins are analog pins. The board also has 4 UART ports that are used for hardware serial connections. The clock speed of the developing board is $16 \mathrm{MHz}$, the speed is given by the integrated crystal oscillator. For the FLASH memory the board has $256 \mathrm{~KB}$ ( $8 \mathrm{~KB}$ are used by the bootloader), for the SRAM memory the board has 8 $\mathrm{KB}$ and for the EEPROM memory the board has $4 \mathrm{~KB}$. The current for the input and output pins is $40 \mathrm{~mA}$ and for the $3.3 \mathrm{v}$ pins is $50 \mathrm{~mA} \mathrm{[6].} \mathrm{The} \mathrm{developing} \mathrm{board} \mathrm{has} \mathrm{an}$ operating voltage of $5 \mathrm{~V}$ and can be powered via the USB or by $7 \mathrm{~V}-12 \mathrm{~V}$ power supply. Arduino Mega 2560 full specifications:

\subsection{ASC712 current sensor}

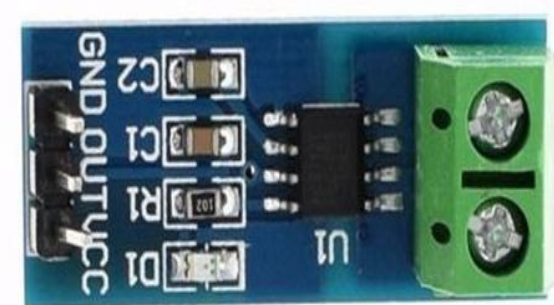

Figure 3 ASC712 current sensor.

The ASC712 linear current sensor (e.g. Fig.3) is a 20A fully integrated current sensor based on the Hall effect, the sensor is suitable for measuring currents up to 20 Amps and has 2.1 KV RMS voltage isolation. The sensor has a integrated current conductor that has very low resistance and is used to calculate and measure the current that is applied to it, the internal conductor resistance is 1.2 $\mathrm{M} \Omega$. For the signal processing the sensor uses a $80 \mathrm{kHz}$ bandwidth that has a low-noise analog signal path. The magnetic hysteresis which influenced the current reading is near zero and the output sensitivity is from $66 \mathrm{mV} / \mathrm{A}$ to $185 \mathrm{mV} / \mathrm{A}$. The sensor is precise with an output error of $1.5 \%$ and a stable offset. ASC712 current sensor full specifications:

- Consumption current: $10 \mathrm{~mA}$;

- LED power indicator;

- 100mV / A output.

- Dimensions: 27 mm x 12 mm x 14 mm.

\subsection{D.C voltage sensor}

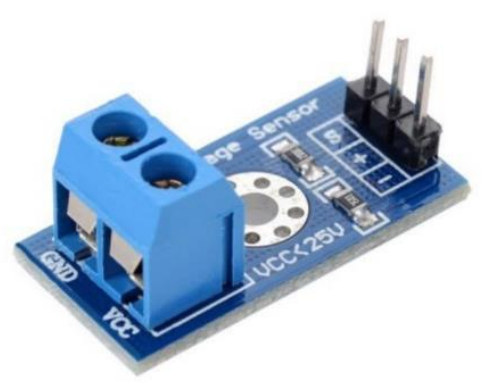

Figure 4 D.C voltage sensor.

The D.C voltage sensor (e.g. Fig. 4) is used to measure, calculate and establish the D.C voltage. The measured device is connected to the sensors input and the measurement depends on the internal voltage divider of the sensor. The sensor has one input connector that has two connections (positive and negative) and one output connector that has three connections (VCC, GND and Signal). The sensor uses a voltage divider that separates into two resistors, one is $30 \mathrm{~K} \Omega$ and the other is $7.5 \mathrm{~K} \Omega$, after a voltage supply is applied the output voltage can be determined by the resistors used by the sensor. The D.C voltage sensor full specifications:

- Voltage Detection Range: $0.02445 \mathrm{~V}$ to $25 \mathrm{~V}$

- Analog Voltage Resolution: 0.00489V

- Dimensions: 28 x 14 x 13 mm

- Weight: $4 \mathrm{~g}$

\subsection{A.C voltage sensor}

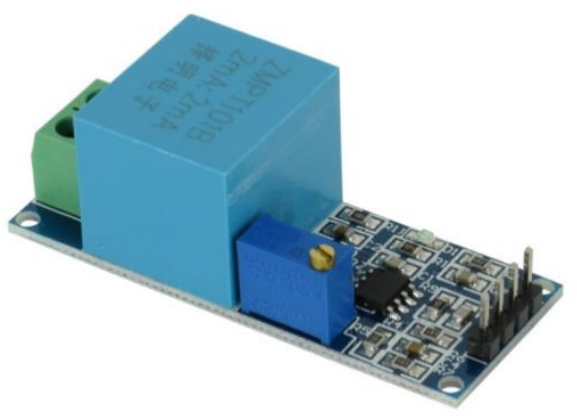

Figure 5 A.C voltage sensor.

The A.C voltage sensor (e.g. Fig.5) is used for measuring the A.C voltage, the sensor uses the principle of capacitive coupling. The capacitive coupling occurs between two circuits whose conductors are at different potentials. Due to the potential difference, an electric field occurs between the conductors. The sensor can be power by $5 \mathrm{~V}$ $30 \mathrm{~V}$ power supply and has a precision transformer and operational amplifier for improved accuracy. The D.C voltage sensor full specifications:

- Maximum measured voltage: 250VAC

- Dimensions: 49.5 x 19.4 mm.

\section{THE ELECTRONIC BOARD}




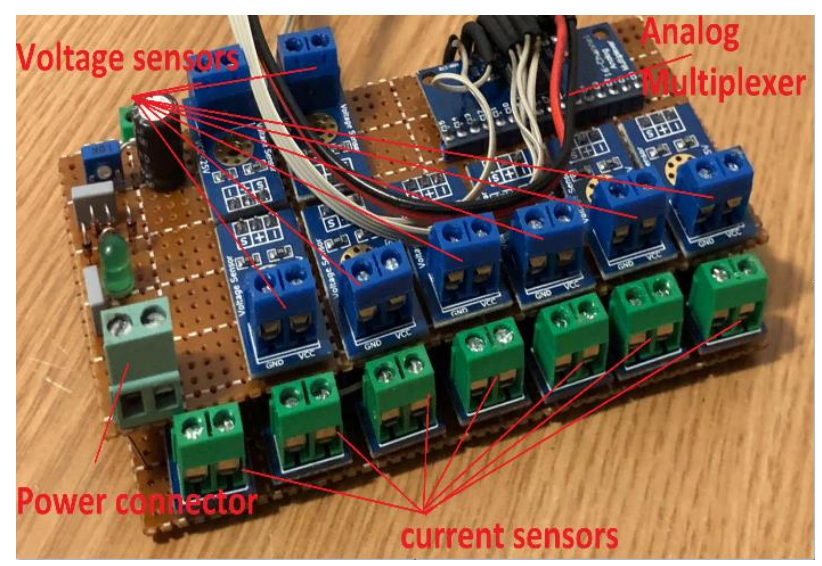

Figure 6 Electronic Board.

The electronic board (e.g. Fig.6) contains the current and D.C/A.C voltage sensors. The signal from the sensors are collected by the analog multiplexer. The board has 10 current sensors, 10 voltage sensors ( 8 D.C voltage sensors and 2 A.C voltage sensors) and 2 analog multiplexers. In the front of the board are positioned 8 voltage sensors and 7 current sensors, the reaming current and voltage sensors are positioned in the back of the electronic board. The analog multiplexers are connected to the Arduino Mega trough a connection cable. The electronic board is power by a $5 \mathrm{~V}$ power supply.

\section{THE FUNCTIONING PRINCIPLE}

The device gives the user the ability to measure and store the current and voltage consumption for any security system. The user can connect 10 security devices (e.g. PIR sensors, video cameras, DVR, control panel). The sensors measure the current and voltage of the connected devices and sends the information to the Arduino Mega 2560 developing board via the analog multiplexers, the Arduino Mega stores the data collected from the sensors on a SD card using the external SD card module and then sends the information via the Ethernet connection on a webpage application that is specially created for viewing the data in real time from any web browser. The user can view the current and voltage consumption for every connected device at the same time thus making the monitoring process of the measured system more efficient.

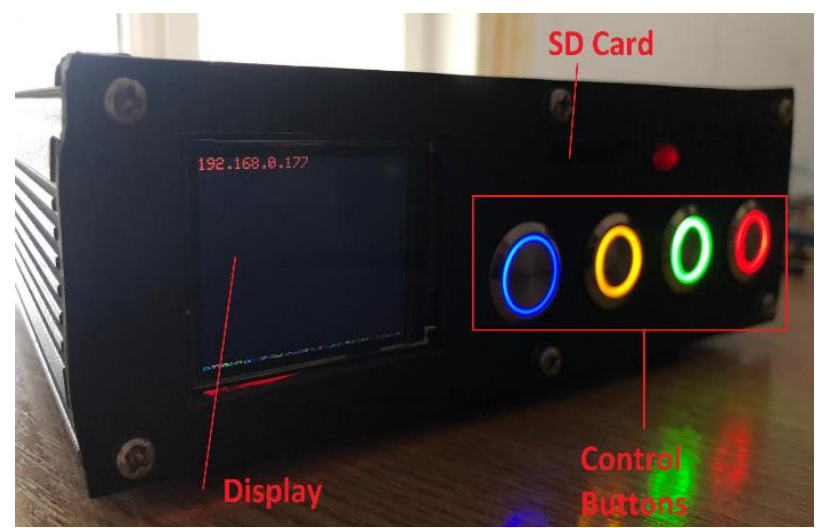

Figure 7 Measuring device.

In Fig.7 we have the device fully assembled. The device uses the 1.8" TFT display for viewing the IP needed for accessing the device by the web browser, the control buttons are used for setting up and configure the device and the SD card space is used to insert the SD card.

\subsection{Web interface}

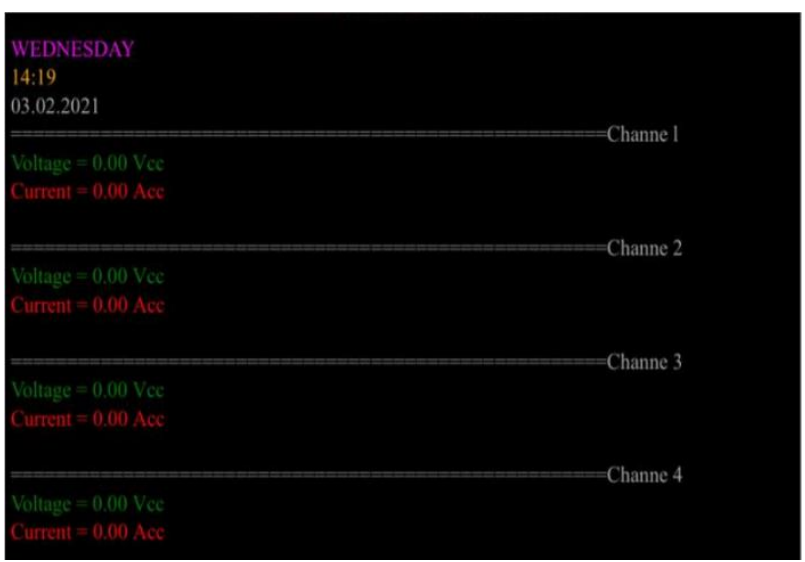

Figure 8. Web application.

The web application (e.g. Fig 8) shows the information collected from all 10 connected security devices measured in real time. The information consists of the current and voltage for each channel (connected device).

\section{RESULTS}

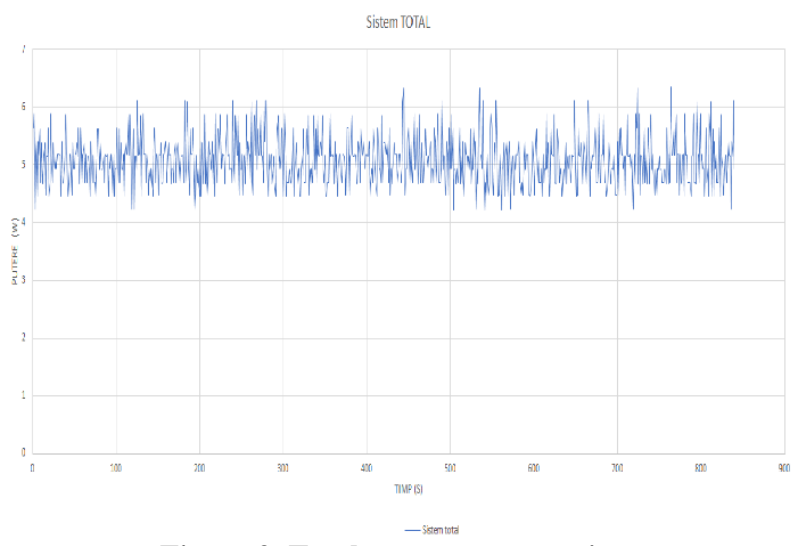

Figure 9. Total power consumption.

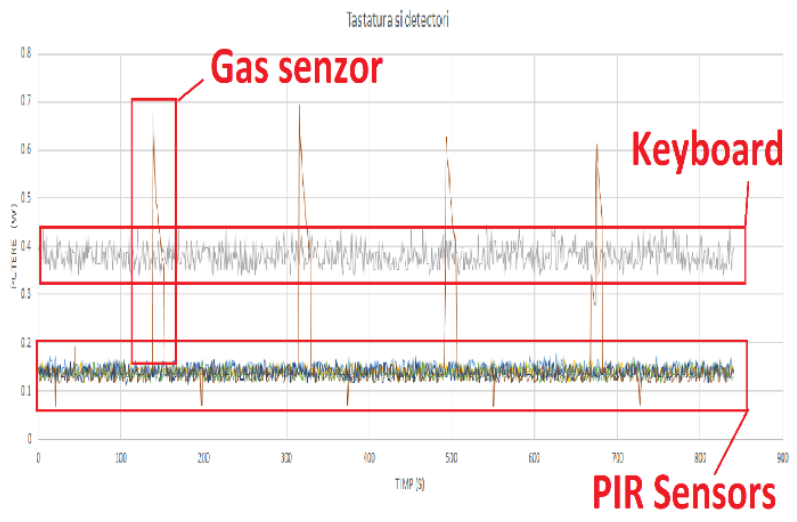

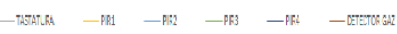

Figure 10. Sensors and keyboard power consumption. 


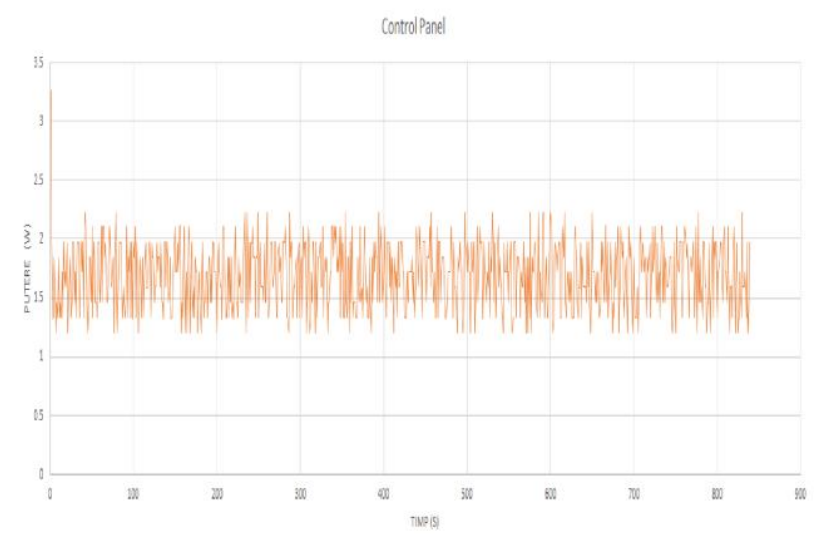

Figure 11. Control panel power consumption.

The control panel, keyboard, PIR sensors have a linear consumption, each varying in its range. It is not very noticeable how each power consumption influences the control panel. The gas detector has a peak value at a time period, which is clearly visible on the graph, this behaviour is normal.

\section{CONCLUSIONS}

The developed device is very useful in obtaining information about the voltage and current consumption of security systems. The 10-channel input of the device can be used to monitor the power consumption for PIR sensors, gas sensors, humidity sensors, keyboard, control panel, DVR and video cameras. The user can use the web application for monitoring the consumption in real time, the web application can be accessed from any web browser by accessing the devices IP. The data obtained from the measurements is stored on a SD card for future analysis. The measurement accuracy of the device is given by the sensors used.

\section{REFERENCES}

[1] R.A. Deshmukh, S. Kamdi, M. Pingle, S. Rajebhosale and A. Bhosale "Intelligent surveillance system using energy efficient intrusion detection and tracking techniques", Proceedings of the 2nd International conference on Electronics, Communication and Aerospace Technology (ICECA 2018) IEEE Conference Record \# 42487; IEEE Xplore ISBN:978-1-5386-0965-1.

[2] L.A. Greening, D.L. Greene and C. Difiglio, "Energy efficiency and consumption - the rebound effect - a survey", Elsevier Energy Policy, Volume 28, Issue 6-7, June 2000, Pages 389-401

[3] E. Diaconu, "Controlul sistemelor electronice", Targoviste, Romania, Valahia University Press, ISBN 978-606-603-170-7.

[4] V. Ion, H.Andrei, E.Diaconu, A. Enescu, I. Udroiu, "Energy Consumption Analysis of Security Systems

for a Residential Consumer", Proceedings of the the 11th international symposium on advanced topics in electrical engineering, Bucharest, Romania, March 28-30, 2019.

[5] John Boxall, Arduino Workshop, No Strach press, 2013.

[6] Jeremy Blum, Exploring Arduino: Tools and Techniques for Engineering Wizardry, John Wiley \& Sons, 2013. 